Rev. Asoc. Esp. NeuropsiQ. 2020; 40(I37): 57-7I

DOI: I0.432 I/So2 I I-57352020000I00004

\title{
Jacques Lacan y la práctica pública en salud mental
}

Jacques Lacan and public practice in mental health

Rafael Arroyo Guillamón

Psiquiatra y psicoanalista. Centro de Salud Mental de Alcobendas, Madrid.

Correspondencia: arroyoguillamon@gmail.com

Recibido: 13/1/2020; aceptado con modificaciones: 17/04/2020

Resumen: Se revisa la relación entre las teorías de Freud y Lacan, a propósito de recientes investigaciones que encuentran notables diferencias entre ellas. A través de la reflexión sobre diversos aspectos del trabajo público en salud mental, y varios ejemplos clínicos, se propone que la enseñanza de Lacan permite un psicoanálisis más acorde a la práctica actual, que en buena parte se ejerce sin las condiciones para las que Freud diseñó su método.

Palabras clave: psicoanálisis, Freud, Lacan, psiquiatría, salud mental.

Abstract: This paper reviews the relationship between Freud's and Lacan's epistemologies, in light of recent research that considers these two major psychoanalytic schools of thought as significantly different. By reflecting on his experience in the public mental health sector, including clinical vignettes, the author proposes that Lacan's teaching allows for a psychoanalytic work better adjusted to the contemporary clinical practice, which typically takes place in conditions that are far from those required by a classic Freudian approach.

Key words: psychoanalysis, Freud, Lacan, psychiatry, mental health. 
"El problema entonces es saber si el psicoanálisis se dejará llevar poco a poco a abandonar lo que por un momento fue vislumbrado o si, por el contrario, manifestará otra vez, dándole nueva vida, su relieve"

$(1, \mathrm{p} .13)$

\section{La RELACión Freud-Lacan}

$\mathrm{L}$

A GRAN COMPLEJIDAd de LA ENSEÑANZA DE JACQUes LACAN ha suscitado muy diversas lecturas e interpretaciones. La mayoría considera su obra como una prolongación de la de Sigmund Freud, si bien con ciertos matices. Algunos psicoanalistas defienden una clara relación entre ambos autores del tipo maestro-discípulo. Otros, según qué noción tomen de Lacan, reconocen en él: a veces una continuidad con Freud, a veces una deformación de sus conceptos y, en otras ocasiones, un cuestionamiento de los mismos (2). Un tercer grupo se niega a pensar la relación Freud-Lacan como una sustitución, ya que ambas teorías resultan inconmensurables; describe entonces un desplazamiento, lo que confirma el avance de la propuesta de Lacan, pero reconoce también una vecindad entre ambos: "No se supera a Freud, no se lo prolonga ni tampoco se lo interpreta: aquí se lo desplaza”, afirma Allouch (3, p.40).

A pesar de las diferencias, todas estas fórmulas identifican a Lacan con un modelo teórico-clínico estrechamente vinculado al maestro de Viena. Lacan, pues, como un satélite que, bien de cerca o más alejado, orbita siempre alrededor del mismo eje: Freud y su paradigma científico. Esto resta originalidad a la propuesta del psicoanalista francés, ya que los conceptos freudianos cobran en sus manos una dimensión muy particular. Además, descuida el beneficio que las ideas de Lacan pueden reportar a la clínica psicoanalítica actual.

Sin embargo, está emergiendo un conjunto de psicoanalistas, en torno al argentino Alfredo Eidelsztein, que propone un Lacan apenas leído: aquel que se apoya en otra epistemología para diferenciarse de Freud e incluso disentir con él; lo cual no carece de consecuencias, puesto que habilita una práctica psicoanalítica con interesantes novedades.

Este trabajo revisa la relación entre las teorías de Freud y Lacan, y las consecuencias clínicas de su delimitación. Especialmente, aprovechando la brecha abierta por estos autores, se propone que la enseñanza de Lacan permite un psicoanálisis más apropiado a la práctica actual, que en buena parte se ejerce fuera del ámbito privado, sin diván y sin otras de las condiciones para las que Freud diseñó su método.

\section{El fAMOSO “RETORNO A FreUd”}

En muchas oportunidades, Lacan manifestó que su enseñanza partía de conceptos de otros autores. No obstante, con frecuencia acababa modificándolos drás- 
ticamente, de modo que adquirían un significado muy diferente al original. La obra del francés está plagada de ejemplos: su aparente filiación con Ferdinand de Saussure se disuelve en la torsión que Lacan realizó de sus leyes del signo lingüístico, apoyándose en autores como Roman Jakobson o Émile Benveniste. Asimismo, su noción de estructura se separó, ya desde su misma gestación, del que pareciera su referencia principal, Lévi-Strauss, gracias a las herramientas de la topología combinatoria ${ }^{1}$. Por último, aunque en su obra saltan a la vista las referencias filosóficas, Lacan se apartó de una visión metafísica del individuo y propuso un sujeto caracterizado por una falta en su ser; esta fue la base su famosa antifilosofia (5).

En cambio, no suele admitirse que Lacan operase del mismo modo con Freud, ni que sus teorías puedan considerarse en algunos puntos muy diferentes. Sobre todo, porque el francés promulgó en múltiples ocasiones su "retorno a Freud"; sentencia que a simple vista traza una clara conexión entre ambos autores. Así pues, los conceptos de Freud que Lacan retoma en su enseñanza (inconsciente, transferencia, pulsión, etc.) tienden a interpretarse bajo la marca originaria de aquel. Por otro lado, los nuevos términos de Lacan (significante, real, goce, etc.) suelen emparentarse con otros de Freud que se consideran casi sinónimos (6-8).

En resumen, muchos autores, aun reconociendo el tratamiento original que Lacan hace de sus fuentes, no se plantean su independencia del padre del psicoanálisis, considerando esta posibilidad, en cierto modo, irreverente ${ }^{2}$.

Con todo, una lectura atenta revela que en cientos de oportunidades Lacan no solo toma a Freud modificando sus términos, sino que abiertamente los critica por equívocos y propone su corrección. Así, respecto a su famoso "retorno a Freud", se pronuncia: "El ideal bien clásico [...] de un retorno a las fuentes no es ciertamente lo que me aferraba. Repensar, ese es mi método" (10). En otra ocasión: "Nuestro retorno a Freud tiene un sentido muy diferente [...] Debe volver a decirse todo sobre otra faz" (11, p. 350). Por último: "Hasta cierto punto yo he vuelto a armar lo que dice Freud. Si hablé de «retorno a Freud» es para que nos convenzamos de cuán cojo es" (12, p. 40).

\section{OTRO PSICOANÁLISIS}

¿Por qué pensar un Lacan diferenciado de Freud? ¿Qué consecuencias tendría para el psicoanálisis actual?

\footnotetext{
${ }^{1}$ Sobre Lévi-Strauss, Lacan afirmó: "Le debo mucho, si no todo. Esto no impide que yo tenga de la estructura una concepción muy diferente de la suya” (4, p. 56).

${ }^{2}$ Por ejemplo, Milner describe que Lacan se mantiene respecto a algunos autores en una posición de inclusión externa. Aun así, afirma: "Para volver a captar el verdadero objeto del psicoanálisis, conviene retornar a Freud" (9, p. 132).
} 
El trabajo de investigación de Eidelsztein se ha orientado en este sentido (1315). Este autor ha examinado una enorme cantidad de citas y referencias de la enseñanza de Lacan. Todas ellas, puestas en serie, proponen una lectura de su obra que no da por sentada su continuidad con Freud. Esta perspectiva rescata del texto de Lacan algunas proposiciones que pudieron pasar inadvertidas. No obstante, suponen importantes consecuencias: a nivel epistemológico y para la práctica del psicoanalista.

En primer lugar, tanto Freud como Lacan divulgaron un psicoanálisis fuertemente vinculado a la ciencia. El primero, con una visión empirista, describió un aparato psíquico ligado a las vicisitudes pulsionales del organismo; sus principales referencias fueron la biología y la física clásica. Lacan, sin embargo, adoptó una posición dialéctica que privilegiaba el lenguaje sobre la realidad material ${ }^{3}$; para ello, se apoyó en la lingüística, la matemática y las físicas relativista y cuántica, ciencias que no se orientaban por los fenómenos observables, sino por la lógica que los subyace.

Por tanto, el psicoanálisis para Lacan no es una experiencia de la que se obtienen datos para descubrir una verdad oculta; consiste en articular, a partir de un discurso, cierto saber, aunque siempre hipotético e incompleto. El psicoanalista, más que traducir un relato, propone una lectura del material y realiza después un esfuerzo de formalización del caso para intervenir sobre él ${ }^{4}$. De este modo, la práctica psicoanalítica corresponde a una función, una lógica con la que operar en muy diversas situaciones. No se define por aquellas condiciones que desde Freud, a pesar de las modificaciones actuales, circunscriben el psicoanálisis a un entorno concreto (la consulta privada), a unos tiempos prefijados (por sesión, entre sesiones o de duración del proceso) o a determinada actitud del terapeuta (un silencio apenas interrumpido por su reveladora interpretación).

Se halla aquí la importancia de diferenciar el modelo de Lacan de la clásica propuesta freudiana: explorar las posibilidades del psicoanálisis en escenarios aparentemente desfavorables, como la sanidad pública.

\section{EL PSIQUI-ANALISTA}

Frecuentemente, el/la psiquiatra que trabaja en el ámbito público considera inadecuado atender los llamados problemas de la vida: rupturas sentimentales, conflictos laborales, dolencias físicas menores, etc. Argumenta que no se debe medicalizar la existencia humana, ni sobrecargar con ello el sistema sanitario. Se traza así una

3 "No hay la más mínima realidad prediscursiva", afirmará (16, p. 44).

${ }^{4}$ Lacan intentó aportar rigor al psicoanálisis formalizándolo en una escritura matematizada, mediante múltiples fórmulas, grafos y esquemas (17). 
distinción —más académica que real— entre salud y enfermedad, entre normalidad y patología o entre problemas y trastornos; lo que no impide que las consultas acaben atravesadas por el malestar cotidiano.

Paradójicamente, la mayor parte de estos casos, supuestamente leves, son etiquetados con el nombre de un trastorno y tratados con medicación, pues el profesional se ve impedido para ofrecer otra alternativa al padecer de su paciente.

Pero es difícil sostener que el Estado deba hacerse cargo por completo o para siempre del sufrimiento humano. He aquí la primera peculiaridad que encuentra un psicoanalista ejerciendo como psiquiatra en un servicio público: si parte de la dificultad en el encuadre clásico está en fidelizar al paciente al método, lejos de esto, el sistema sanitario espera que sean los menos quienes necesiten sus servicios, o que cuando los precisen regresen lo antes posible a valerse por sus propios medios.

Así pues, el psiqui-analista — si se admite el neologismo- debe hacer un cálculo muy preciso: debe resolver qué, cuánto y hasta cuándo trabajar el material que se le ofrece. Quedan excluidas por tanto las condiciones del dispositivo freudiano 5 , y ese modo de dirigir la cura en el que únicamente habla el paciente, pues el analista, que solo interviene para abrir el discurso de aquel, trata de no introducir elementos propios y que la persona aprenda a desenvolverse en los límites de la palabra.

El psiqui-analista, en cambio, trabaja en el acto. Por un lado, abocado a la inmediatez, ya que suele transcurrir bastante tiempo hasta la siguiente sesión (en el mejor de los casos, uno o dos meses), e incluso el paciente puede desaparecer una larga temporada o, a menudo, no regresar. Por otro lado, el profesional trabaja a través de su acción: en palabras de Lacan, "una puntuación afortunada" del discurso del sujeto (18, p. 245), destacando algunos de sus elementos a fin de articularlos de modo diferente para generar cambios en su estructura. Apostará con ello por una lógica particular en cada caso, asumiendo no obstante que esta resulte siempre limitada e incompleta.

\section{Pedir no es Demandar}

Hoy en día, las contingencias de la vida acaban muy habitualmente en la consulta de los médicos de cabecera, cuando no en las urgencias de los hospitales; son los lugares desde donde el paciente accede a los servicios de salud mental, en muchas ocasiones ya con una prescripción farmacológica. La persona acude entonces con la expectativa de que sus síntomas corresponden a desórdenes del cuerpo, y se abandona al prejuicio — casi universal — de que la psiquiatría hace equivalentes subjetividad y cerebro, operando fundamentalmente mediante reequilibrios químicos.

${ }^{5}$ El famoso par asociación libre-atención flotante. 
Muchas de las personas atendidas en un centro de salud mental esperan una solución biológica y ajena a sus posibilidades, solo accesible por el experto: "No sé, usted es el médico", suele ser una respuesta frecuente si en la primera entrevista el psiquiatra pregunta al paciente por su hipótesis acerca de su malestar. Por otra parte, bastantes personas no acuden a petición suya (muchas veces de su entorno) o no atribuyen su padecimiento a algo propio (lo sitúan en la pareja, el jefe, un malestar físico que no controlan, etc.). Así, en la mayoría de casos, no existe demanda alguna de análisis, de terapia ni la más mínima confianza en que hablando pueda aliviarse algo de lo que les aqueja.

Ahora bien, el consultante pide — casi exige — que el profesional decrete en pocos minutos, y con el menor margen de error posible, a qué responde su malestar; y a poder ser, con un nombre de raíz griega que apacigüe sus dudas sobre su origen y revele una eventual solución. Este requerimiento diagnóstico urge incluso para la institución sanitaria, que promueve que los profesionales, ya en el primer encuentro con el paciente, establezcan un juicio clínico y la orientación terapéutica del caso ${ }^{6}$.

También el psicoanálisis se ha visto muy influenciado, desde sus inicios, por el modelo médico, a tenor de la formación como neurólogo de su creador. Es cierto que Freud propuso ańadir al aprendizaje del psicoanalista saberes ajenos a la medicina (19), y que se mostró a favor de la práctica analítica por personal no médico (20). No obstante, su clínica se centró en una investigación semiológica, a fin de reconocer y clasificar signos y síntomas patológicos. Su metapsicología seguía las premisas del neopositivismo de su época.

La práctica de Lacan, en cambio, se inició en la psiquiatría manicomial, más marginal si cabe al discurso médico. Quizá por ello, su enseñanza estuvo sostenida por otras materias que consideró imprescindibles para la práctica analítica: la lingüística, la lógica, la topología y la antifilosofía (5).

\section{EL CAMPO PÚBLICO DE LA PALABRA}

Las diferencias epistemológicas entre Freud y Lacan resultan de gran interés, puesto que podrían conducir a una práctica actual más ventajosa. Hoy, los requisitos clásicos para el trabajo psicoanalítico no son factibles en todos los contextos. Entonces, la consideración lacaniana del inconsciente, no como una verdad oculta, sino como un saber hipotético pero articulado lógicamente, puede ayudar al analista a desempeñar su función en entornos difíciles.

Es crucial que el profesional que ejerce a nivel público advierta que en dichas circunstancias puede hacer un trabajo psicoanalítico serio. Muchos psicoanalistas se resig-

\footnotetext{
${ }^{6}$ Se ha popularizado para ello el término "consultas de alta resolución".
} 
nan ante la limitación que suponen los casos atendidos en este contexto: el reclamo de una solución farmacológica, la vacilación de su compromiso con el tratamiento, el uso a su arbitrio de algunas indicaciones, la notable complejidad de sus síntomas, etc. Se añaden además las condiciones en que se realiza el trabajo (gran número de pacientes en cada jornada, un tiempo de atención insuficiente, etc.) o la interferencia de elementos a priori "poco analíticos", como los efectos de la medicación o las recomendaciones de otros profesionales, a veces con muy diferente criterio o metodología.

Pero, ya solo en sus formas, el psiqui-analista puede utilizar determinados elementos para establecer un marco, en la medida de lo posible, diferente al clásico intercambio entre médico y paciente. Por ejemplo, eliminar la mesa que se interpone entre ambos, prescindir de la bata, no focalizarse en registrar lo que el paciente le cuenta, recibirlo y despedirlo en la puerta estrechándole la mano, etc. Son hábitos que, si bien no son generalizables — cada terapeuta tendrá sus costumbres— ni garantes en sí mismos de un buen proceso, podrían ayudar a privilegiar la palabra ante otras herramientas que se sobrentienden en cualquier psiquiatra.

Puede alegarse que estas medidas forman parte del mínimo trato respetuoso al inicio de cualquier terapia. Sin embargo, no son de uso tan corriente en el ámbito público, donde el encuentro con el profesional adquiere la forma de un intercambio técnico entre quien detenta el saber y quien lo ignora esperando recibirlo externamente. De modo que, en no pocas ocasiones, el paciente se incomoda si se trata de invertir esos roles para favorecer la palabra: bien durante el transcurso de la entrevista, ya que solo esperaba hablar lo justo para que se emita un "veredicto", o bien si hacia el final de la misma se le pregunta cómo ayudarle, siendo el silencio una respuesta más que habitual.

\section{El ASUNTO DEL SUJETO}

Lacan propuso como foco del análisis al sujeto, no a la persona que acude a consulta, con la que suele confundirse. El sujeto es exclusivamente un efecto del lenguaje: un texto elaborado mediante la articulación lógica de ciertos elementos del discurso del paciente que, gracias a la intervención del analista, devienen significantes ${ }^{7}$. Así, se puede leer en Lacan una acepción del término sujeto poco considerada: el asunto, el tema, la cuestión o el problema entramado en los dichos del analista y de la persona que lo consulta. Esta articulación conforma una estructura tal que el cambio de cualquier elemento reorganiza todo el sistema y da lugar a un nuevo sujeto.

Para Lacan toda realidad está antecedida y originada por el lenguaje, incluyendo el cuerpo y sus fenómenos naturales (21). Entonces, el análisis debe diagnosticar

${ }^{7}$ Elementos sin significado en sí mismos, pero susceptibles de combinarse de tal forma que se genere un posible sentido. 
el sujeto y no al sujeto: no se trata de proveer de un diagnóstico al caso, sino de estipular cuál es el tema o asunto en cuestión (22). Para ello, el analista cuestiona el significado ordinario del relato del paciente, tomándolo como una ficción discursiva siempre susceptible de una nueva reorganización. Véanse algunos ejemplos:

Un paciente describió un sueño en el que, sorprendentemente tranquilo, se adentraba en una zona peligrosa que podía comprometer su vida. Avanzada la sesión, dijo que había reincidido, sin saber por qué, en una operación económica de riesgo que ya en el pasado había perjudicado su patrimonio. Posteriormente, mencionó que hacía años había perdido a un bebé en dramáticas circunstancias, al que curiosamente había puesto el nombre de un conocido deportista de una disciplina bastante peligrosa. El analista articuló estos elementos puntuando lo determinante que había sido para él arriesgarse a lo largo de su vida. En el paciente, que en las sesiones previas se había centrado en la ineficacia de los fármacos para calmar su ansiedad, pareció revelarse una nueva perspectiva. Este asunto, en sesiones posteriores, dotó de un posible sentido a diversas anécdotas de su vida en las que se repetían los mismos elementos: su arriesgado sacrificio en busca de un éxito que, si bien nunca alcanzaba, le hacía sentir culpable por haber descuidado a sus seres queridos. Fue un trabajo mínimo, pero suficiente para rebajar su medicación y que accediera a iniciar en el mismo centro un espacio psicoterapéutico.

Otra mujer explicó haber sido desde su infancia la más débil entre sus hermanos. Padeció varias enfermedades que la alejaron largo tiempo de la escuela y obligaron a sus padres a tomar muchas precauciones en su crianza. Las preocupaciones corporales en su vida adulta cobraron tal dimensión que llegó a estar firmemente convencida de padecer una enfermedad mortal, lo que le había procurado varias hospitalizaciones y la prescripción de medicamentos antipsicóticos. En las sesiones sucesivas, el analista recortó y puso en serie algunos puntos de su relato, a propósito de una grave enfermedad que había acabado con la vida de dos de sus hermanos y que terminó padeciendo ella misma, con una actitud mucho más saludable en cada ocasión. Esto construyó, respecto al tema de las enfermedades, un sujeto diferente: aliviado de los autorreproches y la culpa melancólica que arrastraba. Y guio las intervenciones del analista durante siete sesiones (año y medio de tratamiento) en las que la paciente reconsideró varios hitos de su vida, se retiraron todos los fármacos y se interrumpió su seguimiento en salud mental.

\section{EL INDIVIDUO EN CUESTIÓN}

Para Lacan el sujeto resulta de un entrecruzamiento de elementos lingüísticos: los dichos y refranes, los hitos y costumbres, los gozos y sufrimientos que a través de varias generaciones escriben la novela familiar; una narración cuyo guion 
es independiente de quienes la protagonicen, pues los diferentes personajes parecen alternarse, bien en los mismos papeles, o bien en sus opuestos. En términos geométricos, si se imagina al sujeto como una estructura compuesta de varias piezas, lo más importante sería el orden que las mantiene unidas; siempre que se respete la misma organización de elementos, cambiar el tamaño o el material de las piezas no alteraría la esencia de la estructura, solo daría lugar a otra equivalente.

Esta perspectiva no limita el análisis al trabajo individual con el paciente; permite incorporar otros elementos (otros capítulos de la novela o piezas de la estructura) que ayuden a formular la pregunta que define al sujeto/problema.

Una mujer describió que su hijo había sido un niño inquieto, inmaduro e irresponsable. Actualmente, el muchacho solo podía calmar su ira fumando cannabis, lo que le había acarreado problemas escolares y con la justicia. Su madre exigía una prueba médica de su probable "hiperactividad", una pastilla para corregir su comportamiento y un informe que lo protegiese legalmente, ya que su incorregible conducta no tardaría mucho en hacerle reincidir. En entrevistas con ambos durante aproximadamente un año, se vio que esta familia estuvo marcada por la mala relación entre los abuelos maternos, la rebeldía y el coqueteo con las drogas de la madre y su posterior matrimonio con el padre, una persona inestable del que se separó traumáticamente siendo el chico un infante. Tanto el muchacho como un hermano habían presentado problemas de conducta, consumo de sustancias e incluso un intento de quitarse la vida. Tras la identificación de estas repeticiones en el asunto familiar, el muchacho se mostró más colaborador, comunicativo y completó sus estudios. Esto dio por buena la interrupción de un tratamiento para el que, finalmente, no fue necesario añadir medicación.

Otra chica relató en su primera entrevista que el capricho de algunos profesores le había impedido varias veces completar sus estudios. De forma similar, su madre —incorporada en la segunda parte de la sesión — explicó haber dejado su trabajo por sentirse repetidamente perjudicada por sus responsables. Desde hacía años, madre e hija compartían diversas afecciones físicas aparentemente inexplicables. Además, esta madre y su progenitora (abuela de la paciente) habían vivido relaciones de pareja insatisfactorias con hombres "mueble" totalmente al margen de la vida familiar, volcándose en el cuidado de algún pariente enfermo. Dada esta forma familiar de relación, en la que el otro perjudica, no pinta nada o es un objeto a cuidar, la muchacha nunca había establecido amistades verdaderas ni, menos aún, flirteo alguno; permanecía como partenaire de una madre francamente melancólica y empezaba a desarrollar quejas depresivas del mismo tipo.

¿Quién debe considerarse aquí el sujeto de la intervención analítica? ¿Cada una de ellas individualmente, con sus diferentes inconscientes y conflictos? ¿ $\mathrm{O}$ se puede trabajar con ambas para construir una fórmula con la que deducir la ley 
general que determine — con distintas variables en cada generación — el resultado de la función familiar?

\section{UN SUJETO DE OTRO TIEMPO}

En la construcción del sujeto se condensan diferentes momentos, recuerdos o personas que el paciente de inicio presenta como hechos aislados y, sobre todo, acontecidos realmente en su pasado. Sin embargo, en la medida en que estos sucesos son transformados en hechos de discurso por efecto del decir del Otro ${ }^{8}$, no solo cobran una luz diferente (no se trata solo de resignificar el pasado), sino que la persona puede verificar que quizá, tal y como ella los consideraba, nunca ocurrieron. El tiempo para Lacan pierde así su dimensión cronológica y lineal (la famosa flecha que avanza desde el pasado hacia el futuro) tornándose un tiempo reversivo o circular: en él es posible la anterioridad lógica del futuro, es decir, que acontecimientos cronológicamente posteriores puedan — por extraño que parezca a nuestro sentido común— cambiar el pasado (23). Así, una persona puede comprobar durante su tratamiento que aquellas certezas que sostenían "la verdad" de su vida corresponden a una ilusión narrativa.

Esta perspectiva del tiempo, ahistórica y estructural, es especialmente útil para el trabajo público en salud mental, en el que se dispone de pocos minutos en cada sesión y de una frecuencia de citas claramente insuficiente. Entonces, el sujeto, como articulación de elementos lingüísticos, requiere de un tiempo lógico, no cronológico; lo que permite un trabajo razonable sin necesidad de largas sesiones o incluso transcurriendo semanas entre una y otra. Todo ello siempre que no se aborte la emergencia del discurso del Otro con recursos que, si bien se necesitan en ocasiones, en otras buscan solo el alivio inmediato, situando la esperanza por mejorar en medidas externas: la medicación, las ayudas sociales, las pautas de conducta, la bondad del terapeuta, etc. Dos ejemplos:

Tras una única sesión, la llamada de teléfono de una paciente sorprendió al analista: "Cuando estuve con usted —explicó la mujer— volví a casa y le dije a mi marido que el médico me había ordenado que me separase; ahora estoy feliz, no necesito más ayuda". El terapeuta no recordaba haber proferido tal indicación. Más bien, tras un largo rato en que ella había descrito los años de matrimonio desdichado, siendo que todo su entorno (hasta su anterior psiquiatra) le recomendaba separarse, le había preguntado por qué motivo seguía unida a él. La pregunta devolvía a la paciente su propia demanda en forma invertida desde el Otro, aportando una nueva óptica que la ayudó a tomar una decisión que, sin duda, ya había valorado muchas veces sin atreverse a efectuarla.

\footnotetext{
${ }^{8}$ Para Lacan, el Otro es el conjunto de los términos del lenguaje; todos ellos potencialmente articulables según leyes lógicas en un tema o asunto. Corresponde pues a una función, no a una persona, si bien puede ser encarnado puntualmente por determinados personajes de la historia del individuo.
} 
Otro señor consultó muy asustado por un fuerte ataque de pánico que había experimentado en un balcón sin aparente motivo, puesto que era dichoso en todos los aspectos de su vida. Lo sucedido le había dejado un miedo atroz a que se repitiera la crisis, que le impedía utilizar ascensores o subir a determinadas alturas. Valió una pequeña coincidencia señalada por el analista, a saber, que el episodio sucedió en el mismo mes en que años antes había fallecido su madre, para que la persona hablase largo y tendido sobre el dolor que le había causado esta pérdida. Fue él mismo quien, en la segunda visita, sintiéndose muy mejorado, solicitó concluir la ayuda.

\section{¿ANÁLISIS INTERMINABLE O INTERMITENTE?}

Resultaría oportunista concluir que, en encuentros tan breves como los anteriores, fue solo la intervención del analista la que causó la mejoría del paciente. Es probable que se hubiera producido igualmente con otros profesionales o mediante diferentes enfoques terapéuticos. Lo que aporta en este caso su especificidad al método psicoanalítico es la interpretación de esos acontecimientos como causados - y susceptibles o no de modificarse- por el lenguaje.

Así pues, los psicoanalistas en este ámbito no hablan de un fin de análisis; si acaso de un proceso intermitente, en tanto se desarrolla en distintas etapas. Es posible también que ocurra al revés: en ocasiones el tratamiento público puede ser un paréntesis en el que el paciente decide retomar una terapia iniciada fuera de allí.

Una mujer con varios períodos depresivos durante su vida había concluido su análisis a nivel privado con la siguiente fórmula: el cáncer digestivo que había superado simbolizaba para ella haber digerido bien "toda la mierda acumulada" desde la infancia por la conflictiva relación con su madre. Los síntomas depresivos por los que ahora consultaba no guardaban, aparentemente, relación con ese asunto que parecía olvidado. Algunas entrevistas en la consulta pública sirvieron para relacionarlos con la inseguridad que manifestaba en la crianza de su hija adolescente, que la trasladaban a los problemas con sus progenitores cuando ella tenía su edad. Dada su mejoría, pudo iniciarse la retirada de los fármacos que le había prescrito su médico de cabecera y la paciente decidió reanudar su análisis.

Intervenciones tan recortadas requieren una práctica psicoanalítica alejada de la clásica neutralidad. Hoy en día, la ciencia descarta que la realidad pueda observarse neutralmente: el observador participa necesariamente en su construcción, al no poder prescindir de presupuestos teóricos cuando aborda lo observado. Más aún, ni siquiera es posible situar exactamente un objeto en la realidad salvo en términos de probabilidad?.

\footnotetext{
${ }^{9}$ Según el principio de incertidumbre de la física cuántica, ya trabajado por Lacan (24).
} 
De igual modo, el sujeto del psicoanálisis no puede describirse objetivamente excepto mediante intervenciones estimatorias, que aspiran a ser verosímiles pero no exactas. En estas el psicoanalista, al seleccionar ciertos elementos del discurso del paciente, siempre deja entrever su deseo. Así, si bien Freud abogaba por un terapeuta neutral, Lacan contaba con el deseo del analista, que opera, como en el científico, modificando su objeto de observación (25); lo que pone en cuestión la actitud silenciosa que muchos psicoanalistas siguen practicando.

Por tanto, el psicoanálisis —y menos en el entorno público— no puede ser neutral, y su objeto de estudio, el sujeto, no se encuadra en unos parámetros fijos, ni de tiempo ni de espacio.

\section{UN ESPACIO TRANSUBJETIVO}

Lacan apostó por una idea de espacio que no se corresponde con la geometría euclidiana que se enseña en la escuela; es decir, un lugar fijado por ciertas coordenadas de situación. Siguiendo los avances de la matemática y la física relativista, indicó que espacio y tiempo no son absolutos ni separados, sino que existe una continuidad entre ambos, de forma que en la clínica aparecen superpuestos.

El sujeto no existe solo en el lapso entre el nacimiento y la muerte del individuo; lo precede antes de su alumbramiento y lo trasciende tras fallecer. Del mismo modo, la subjetividad no es particular de una persona: no se ciñe al espacio limitado por su cuerpo ni posee un interior y un exterior bien diferenciados. Lacan recurrió a múltiples fórmulas lingüísticas y matemáticas para expresar que lo más nuclear del sujeto no es una consistencia propia, sino un vacío originado en el lugar del $\mathrm{Otro}^{10}$. El sujeto se revela pues en el intersticio de muy diferentes momentos y lugares, en los que la persona quizá nunca estuvo ni estará físicamente, pero, en la medida en que sí lo hicieron las palabras, los sucesos, los deseos y fantasías familiares y culturales, resulta causado por dicho discurso.

Sobre las consecuencias clínicas de esta concepción, ya se ha adelantado que el psicoanalista en la consulta pública incorpora frecuentemente a los familiares del paciente. Asimismo, resulta habitual que el análisis precise integrar información de otros estamentos profesionales (psiquiatría, psicología, enfermería, trabajo social, etc.) y de diversos espacios terapéuticos o institucionales (psicoterapia grupal, sesiones clínicas, supervisiones, etc.). Estos encuadres constituyen, al fin y al cabo, espacios discursivos donde puede precipitar el asunto/sujeto más allá de la sesión individual.

${ }^{10}$ Véanse algunos de sus conceptos (deseo del Otro, goce del Otro, inmixión de otredad, extimidad, etc.), así como sus comparaciones del sujeto con figuras topológicas (los dos toros entrelazados, la banda de Möbius, la botella de Klein, el cross-cap, etc.). 
Fue el caso de una muchacha, gravemente depresiva, que tras un año de tratamiento individual poco fructífero solicitó interrumpirlo, pero no se opuso a que su madre iniciase en el mismo centro un grupo de terapia para otros pacientes y sus familiares. Meses después la paciente, que llegó a acudir en una ocasión a dicho grupo, pidió retomar su proceso individual que continúa actualmente.

Para otra mujer, algunas entrevistas junto a su esposo e hijos permitieron reflexionar sobre sus inexplicables ataques de ira, por los que había sido diagnosticada de bipolaridad y tratada ineficazmente con diversos fármacos. Su proceso continuó mediante una psicoterapia de grupo donde se escenificaban estas situaciones con otras personas, además de sesiones individuales en las que pudo construir hipótesis sobre las raíces infantiles y familiares de su comportamiento; esto le procuró una importante mejoría y permitió suspender casi toda su medicación.

En otro paciente, fue el trabajo del psicoanalista con sus padres, mediante contactos telefónicos y en el mencionado grupo multifamiliar, lo que sostuvo el proceso terapéutico mientras él permanecía recluido en su domicilio sin apenas contacto social. Un trabajo nada desdeńable en una persona que, a pesar de sus grandes dotes intelectuales, cada tanto tiempo se sumía en intensos estados melancólicos que ponían incluso en riesgo su vida.

\section{Conclusiones}

La mayoría de psicoanalistas considera la obra de Jacques Lacan como una continuación de la de Sigmund Freud. Este fenómeno, basado en una interpretación literal de su "retorno a Freud", descuida las posibles diferencias entre ambos autores.

Recientes investigaciones de un grupo de psicoanalistas, encabezado por Alfredo Eidelsztein, examinan al detalle la enseñanza de Lacan, identificando notables diferencias con Freud. Su lectura se basa en las divergencias epistemológicas entre ambos autores, que, usando conceptos distintos del tiempo, el espacio, la materia y la energía, se sitúan en paradigmas científicos diferentes. El inconsciente y el aparato psíquico trabajados por Freud quedarían así distinguidos de la noción de sujeto en Lacan.

Esta perspectiva facilita una reflexión crítica sobre algunos aspectos del psicoanálisis actual, como su relación con la ciencia. Pero, sobre todo, extrae de la obra de Lacan conclusiones novedosas en cuanto a su práctica: especialmente, sobre las posibilidades del psicoanálisis en ámbitos poco propicios, como la sanidad pública.

El contexto de la atención pública en salud mental dificulta realizar tratamientos según los términos con que Freud inauguró el psicoanálisis. Sin embargo, para Lacan, el trabajo del psicoanalista corresponde a una función, una lógica con la que operar en muy diversos escenarios, no necesariamente vinculada al clásico encuadre ni a sus requisitos técnicos. Esto permite una aplicación de su método con 
menos restricciones en su práctica, para extender su beneficio terapéutico a sectores más amplios de la población. Además, el autor francés defendió un psicoanálisis argumentado, coherente y en permanente diálogo con otras disciplinas científicas. Su conceptualización rigurosa y un esfuerzo por formalizarlo podrían combatir los prejuicios que lo excluyen aún de muchas instituciones sanitarias.

\section{Bibliografía}

(1) Lacan J. El Seminario 2: El yo en la teoría de Freud y en la técnica psicoanalítica (1954-1955). Buenos Aires: Paidós, 2008.

(2) Schejtman F. Una introducción a los tres registros. En: Schejtman F (compilador). Psicopatología clínica y ética: de la psiquiatría al psicoanálisis. Olivos: Grama Ediciones, 2015; p. 385-447.

(3) Allouch J. Freud desplazado. En: Peretti H (dir.). Littoral, textos de psicoanálisis: Lacan censurado. Córdoba, República Argentina: La Torre Abolida, 1986.

(4) Lacan J. Massachusetts Institute of Technology: 2 de diciembre de 1975. En: Conferencias y charlas en universidades norteamericanas [Internet]. Disponible en: https://www.lacanterafreudiana.com.ar/2.5.1.26\%20\%20\%20\%20CONFERENCIAS\%20Y\%20CHARLAS\%20EN\%20UNIVERSIDADES\%20NORTEAMERICANAS,\%201975.pdf.

(5) Lacan J. Quizás en Vincennes. En: Otros escritos. Buenos Aires: Paidós, 2012; p. 333-335.

(6) Eidelsztein A. La gran crisis silenciada del psicoanálisis [Internet]. 2019. Disponible en: https://www.eidelszteinalfredo.com.ar/wp-content/uploads/2019/02/la-gran-crisis-silenciada.pdf

(7) Krymkiewicz M. Modelos energéticos en psicoanálisis. Diferencias entre Sigmund Freud y Jacques Lacan. Revista Affectio Societatis (Medellín, Colombia) 2015; 12(22): 59-69.

(8) Bonoris B. La invención lacaniana del concepto de goce. Revista Affectio Societatis (Medellín, Colombia) 2016; 13(25): 119-144.

(9) Milner JC. La obra clara: Lacan, la ciencia, la filosofía. Buenos Aires: Paidós, 1996.

(10) Lacan J. El Seminario 13: El objeto del psicoanálisis (1965-1966). Clase del 1 de junio de 1966. Inédito.

(11) Lacan J. De un designio. En: Escritos 1. Madrid: Biblioteca Nueva, 2013; p. $347-$ 350.

(12) Lacan J. Apertura de la sección clínica. En: Ornicar? III. Barcelona: Pettel, 1981.

(13) Eidelsztein A. Otro Lacan: Estudio crítico sobre los fundamentos del psicoanálisis lacaniano. Buenos Aires: Letra Viva, 2015.

(14) Eidelsztein A. No hay que salvar a Freud [Internet]. 2019. Disponible en: https:// www.eidelszteinalfredo.com.ar/wp-content/uploads/2019/01/NO-HAY-QUESALVAR-A-FREUD.pdf 
(15) Eidelsztein A. Seminario “Desambiguar Freud de Lacan”, Clase 1 [Internet]. 2020. Disponible en: https://www.youtube.com/watch?v=z94PI11DJNg\&t=4s

(16) Lacan J. El Seminario 20: Aún (1972-1973). Buenos Aires: Paidós, 2008.

(17) Guitart R. Evidencia y extrañeza: matemática, psicoanálisis, Descartes y Freud. Buenos Aires: Amorrortu, 2003.

(18) Lacan J. Función y campo de la palabra y el lenguaje en psicoanálisis. En: Escritos 1. Madrid: Biblioteca Nueva, 2013; p. 231-309.

(19) Freud S. ¿Debe enseñarse el psicoanálisis en la universidad? En: Obras completas, volumen 17 (1917-1919). Buenos Aires: Amorrortu, 2007; p. 165-171.

(20) Freud S. ¿Pueden los legos ejercer el análisis? En: Obras completas, volumen 20 (1925-1926). Buenos Aires: Amorrortu, 2008; p. 165-244.

(21) Eidelsztein A. El origen del sujeto en psicoanálisis: del Big Bang del lenguaje y el discurso. Buenos Aires: Letra Viva, 2018.

(22) Eidelsztein A. Diagnosticar el sujeto. Revista Imago Agenda [revista electrónica] 2003; 73. Disponible en: http://www.imagoagenda.com/articulo.asp?idarticulo=704

(23) Ludueña F. El tiempo en Minkowski y en Lacan [Internet]. 2012. Disponible en: https://www.aacademica.org/000-072/111.pdf

(24) Goldsmidt J. Psicoanálisis y física cuántica: ¿`Toda ciencia forcluye la verdad? [Tesis de licenciatura]. Buenos Aires: Facultad de Psicología, Universidad de Buenos Aires, 2018.

(25) Carrere P. Transferencia vs. Sujeto supuesto saber [Internet]. 2019. Disponible en: https://www.youtube.com/watch?v=QMk_yHasQG4\&list=PLZe1fZwZmCuDFWWDUQVdBbYPbktRx2o5k\&index $=10$ 